\title{
Tracking and Quantitation of Retroviral- Mediated Transfer Using a Completely Humanized, Red-Shifted Green Fluorescent Protein Gene
}

BioTechniques 22:162-167 (January 1997)

R.R. Muldoon, J.P. Levy, S.R. Kain $^{1}$, P.A. Kitts ${ }^{1}$ and C.J. Link Jr.

Human Gene Therapy Research Institute, Central Iowa Health Systems, Des Moines, IA, and ${ }^{1}$ CLONTECH Laboratories, Palo Alto, CA, USA

\begin{abstract}
We have developed murine retroviral vectors $(R V s)$ containing an optimized green fluorescent protein (GFP) gene to study retroviral gene transfer and expression in living cells. We used the codon " $\mathrm{hu}$ manized", "red-shifted" GFP gene, hGFPS65T, a gain of function variant of the wild-type GFP from the jellyfish Aequorea victoria. We cloned the hGFP-S65T gene into the RV plasmid pLNCX ( $p L N C h G 65 T$ ). A stable amphotropic $R V$-producer cell line (VPC), designated LNChG65T VPC, was generated that exhibited bright fluorescence in greater than $95 \%$ of the cells. Human A375 melanoma cells and IGROV ovarian carcinoma cells transduced from LNChG65T VPC demonstrated high levels of fluorescence. The expression of a single integrated hGFP-S65T gene in eukaryotic cells provides a powerful tool to study gene transfer, expression and functional studies in vitro and in vivo.
\end{abstract}

\section{INTRODUCTION}

Human gene therapy involves the transfer of therapeutic genes into living human cells $(4,12,15)$. Murine replication-defective retroviral vectors (RVs) are used as the gene transfer vehicle in two-thirds of the current gene therapy clinical protocols (11). To study gene transfer and expression in vivo and in vitro, we previously developed an RV using a markedly improved green fluorescent protein (GFP) gene containing a red-shifted, partially humanized GFP (10). We now report using a red-shifted, completely humanized GFP gene, the hGFP-S65T gene, which has 169 codon changes compared to 88 in our prior report. Efficient fluorescence of this GFP protein allowed direct detection of gene transfer and activity in living mammalian cells.

GFP is a 730-bp gene cloned from the jellyfish Aequorea victoria that encodes a 27-kDa protein (14). Upon exposure to blue light $(450-490 \mathrm{~nm})$, the protein emits a vibrant green fluorescence. Wild-type GFP expression has been applied in a number of eukaryotic and prokaryotic systems $(1,2,9,13,16)$. Heim and colleagues developed an improved GFP gene variant (S65T) with a "red-shifted" excitation maximum, which has a fluorescent intensity approximately five times stronger than wild-type GFP and fourfold faster fluorophore oxidation (8). Zolotukhin et al. partially "humanized" the "red-shifted" mutant by introducing 92 silent mutations that alter the codons to those more commonly used in human genes (17). The new variant $h$ GFP-S65T gene has even greater modifications (7). Our data with this novel GFP variant gene in human cells demonstrate improved fluorescence compared to previously available GFP variants.

\section{MATERIALS AND METHODS}

\section{Cell Culture}

The following cell lines were used: A375, a human melanoma cell line (ATCC, Rockville, MD, USA); IGROV,

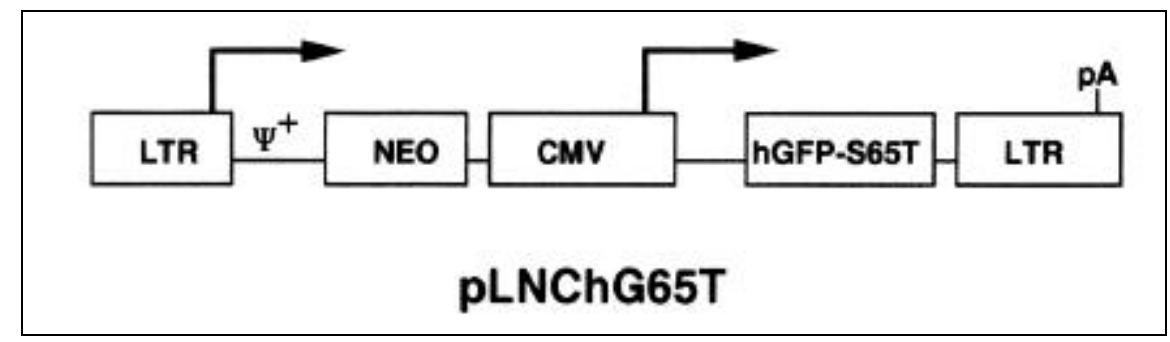

Figure 1. Retroviral construct containing red-shifted, humanized green fluorescent protein. The $h G F P-S 65 T$ gene from phGFP-S65T was cloned into the retroviral vector LNCX to produce pLNChG65T. LTR, long terminal repeat; $\mathrm{pA}$, polyadenylation signal; arrows indicate transcriptional start sites; $\Psi^{+}$indicates the presence of viral packaging sequence; hGFP-S65T, humanized, red-shifted GFP. 


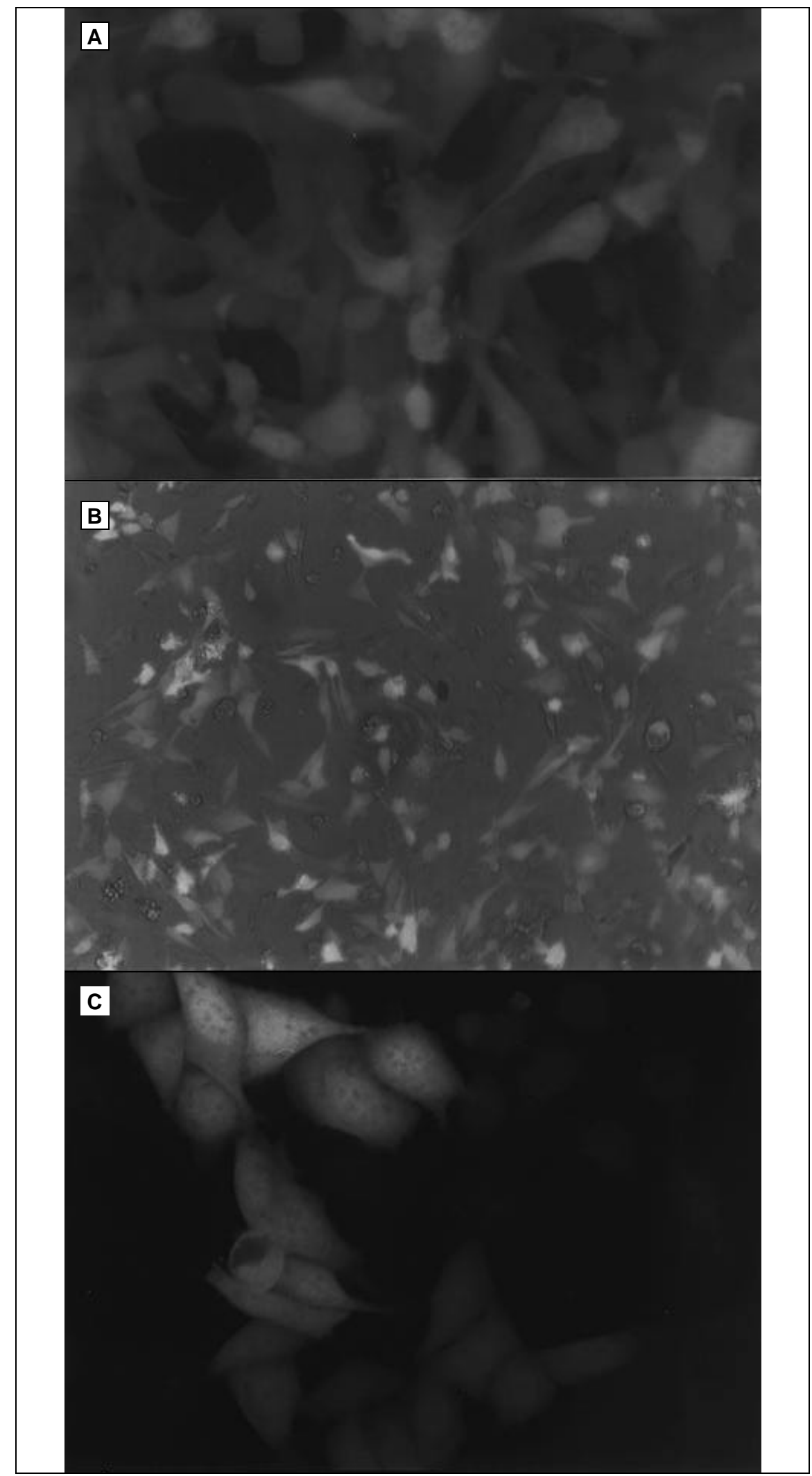

Figure 2. Detection of hGFP-S65T activity in transduced cell lines. A) PA317-LNChG65T cells (40X magnification). B) A375-LNChG65T cells $72 \mathrm{~h}$ after transduction with background light (10× magnification). C) IGROV-LNChG65T cells 5 days after transduction and G-418 selection for 4 days $(40 \times$ magnification). an ovarian carcinoma cell line (kindly provided by Patrick Hwu, National Cancer Institute); PA317, a murine amphotropic, RV-packaging cell line (kindly provided by A.D. Miller, University of Washington); and GPE86, a murine ecotropic, RV-packaging cell line (kindly provided by Arthur Banks, Columbia University). Cells were grown in RPMI supplemented with $10 \%$ fetal calf serum (FCS) (both obtained from Life Technologies, Gaithersburg, MD, USA) in monolayers at $37^{\circ} \mathrm{C}$ and $5 \% \mathrm{CO}_{2}$. All cells were passaged and harvested by standard trypsin (Life Technologies) digestion at $37^{\circ} \mathrm{C}$. Cells were routinely passaged at $80 \%-$ 90\% confluence.

\section{Construction of GFP Retroviral Vector}

Plasmid phGFP-S65T was obtained from CLONTECH Laboratories (Palo Alto, CA, USA). Cloned construct plasmid DNA was transformed into MAX EFFICIENCY DH5 $\alpha^{\mathrm{TM}}$ Competent Cells (Life Technologies), and colonies were grown on L-broth supplemented with ampicillin $(50 \mu \mathrm{g} / \mathrm{mL})$ plates (LB/ AMP). Positive colonies were grown in LB/AMP broth, and plasmid DNA was isolated using the plasmid kit from Qiagen (Chatsworth, CA, USA). Plasmid phGFP-S65T was restriction-digested with HindIII and NotI to obtain the 750-bp hGFP-S65T open reading fragment, which was then gel-isolated (Jetsorb $^{\circledR}$; Genomed, Raleigh, NC, USA). Plasmid pLNCX was restriction-digested with HindIII and HpaI. The hGFPS65T fragment was then ligated into linearized pLNCX, followed by treatment with a Klenow enzyme to remove the NotI overhang. This $3^{\prime}$ end was then ligated to the HpaI site to produce plasmid pLNChG65T.

\section{Construction of the LNChG65T Vector-Producer Cell Line}

GPE86 cells were plated in a 6-well dish $\left(\right.$ Falcon $\left.{ }^{\circledR}\right) 24$ h before transfection. Cells that were at $30 \%-50 \%$ confluence were transfected with $5 \mu \mathrm{g}$ of pLNChG65T plasmid DNA and $15 \mu \mathrm{L}$ of DOTAP reagent (Boehringer Mannheim, Indianapolis, IN, USA) according to the manufacturer's protocol. After $12 \mathrm{~h}$, the cells were rinsed, and $1 \mathrm{~mL}$ 
of fresh medium was placed in each well. RV supernatant was collected 24 $\mathrm{h}$ later and filtered $(0.45 \mu \mathrm{m}$; Corning Costar, Cambridge, MA, USA); then $10 \mu \mathrm{g} / \mathrm{mL}$ of protamine sulfate solution were added. This supernatant was transferred to a 6-well dish containing
PA317 cells that were at $30 \%-50 \%$ confluence. After $24 \mathrm{~h}$, both cell lines were trypsin-digested and transferred to a $10-\mathrm{cm}$ tissue culture dish (Falcon). Cells were selected in $1 \mathrm{mg} / \mathrm{mL}$ G-418 (Genteticin ${ }^{\circledR}$; Life Technologies) for 10-14 days.

\section{Transduction of A375 and IGROV Human Tumor Cell Lines}

LNChG65T VPC cells were grown to $80 \%-90 \%$ confluence in Nunclon ${ }^{\mathrm{TM}}$ T-175 flasks (Allegiance Health Care, McGaw Park, IL, USA). The medium

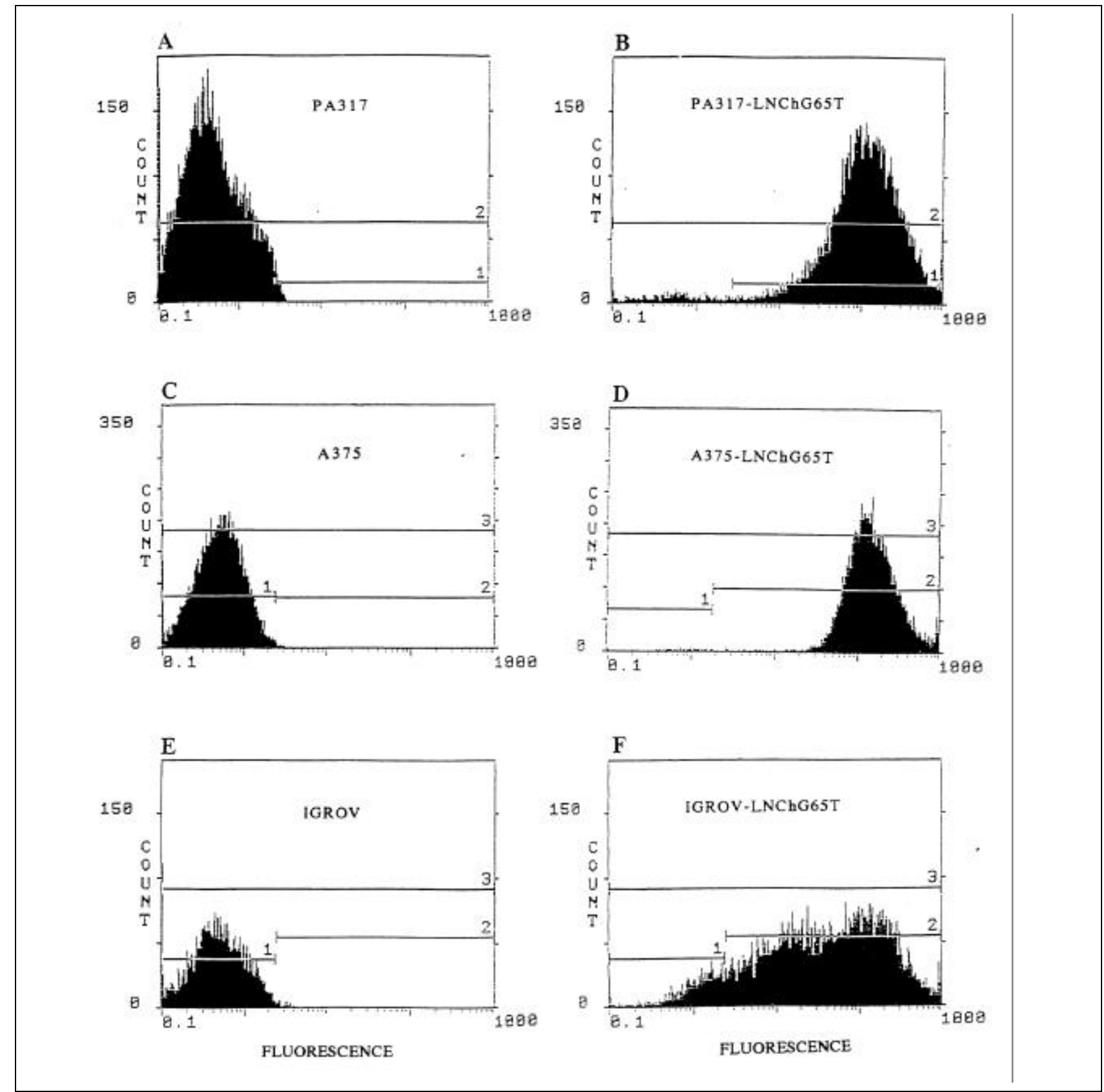

Figure 3. FACS analysis of hGFP-S65T-transduced PA317 vector-producer cells and human melanoma cells. A) PA317 packaging cells are $98.4 \%$ negative (bar 2). B) PA317-LNChG65T cells are 95.8\% positive (bar 1). C) A375 cells are 99.5\% negative (bar 1). D) A375-LNChG65T cells are 98.7\% positive (bar 2). E) IGROV cells are $98.9 \%$ negative (bar 1). F) IGROV-LNChG65T cells are $89 \%$ positive (bar 2). All FACS analyses used the FL1 emission channel used to monitor green fluorescence. Count: Cell number counted at given fluorescence intensity; log scale represents the mean intensity of green fluorescence detected at $525 \mathrm{~nm}$. 
was replaced with $20 \mathrm{~mL}$ of fresh medium, and $24 \mathrm{~h}$ later the RV supernatant was collected, filtered and supplemented with $10 \mu \mathrm{g} / \mathrm{mL}$ protamine sulfate. A375 and IGROV cells were plated onto $10-\mathrm{cm}$ tissue culture dishes and in 6-well dishes containing sterile coverslips. Ten milliliters of the supernatant were added to the culture dishes, and 2 $\mathrm{mL}$ were added to each well of the 6well plates. The supernatant was removed $24 \mathrm{~h}$ later and replaced with fresh medium containing G-418 (1 $\mathrm{mg} / \mathrm{mL}$ ) and selected for 7-14 days.

\section{Fluorescent Detection of GFP- Expressing Cells}

The hGFP-S65T-expressing cells were visualized with a Nikon Labophot-2 ${ }^{\mathrm{TM}}$ fluorescent microscope (Melville, NY, USA). The filter cube used in the microscope was the FITC dichroic filter set (excitation at 450-490 $\mathrm{nm}$ and emission at $520 \mathrm{~nm})$. The coverslips from the 6-well dishes were inverted and placed on a glass slide for viewing. Photographs were taken using the Microflex $^{\mathrm{TM}}$ AFX-DX systems (Nikon).

\section{Fluorescence-Activated Cell Sorter (FACS) Analysis of LNChG65T- Transduced Mammalian Cells}

Cytometry of transduced cells was performed on an EPICS ${ }^{\circledR}$ Profile II Analyzer (Coulter, Miami, FL, USA) with an excitation source of $488 \mathrm{~nm}$. Cells were analyzed using a 525-nm bandpass filter set (Part No. 814036; Coulter). Cultures of cells that were $80 \%-90 \%$ confluent were trypsin-digested, washed with RPMI with $10 \%$ FCS and resuspended at a concentration of approximately $1 \times 10^{6}$ cells $/ \mathrm{mL}$. All FACS analyses used the FL1 emission channel to monitor green fluorescence (normally an FITC monitor).

\section{RESULTS}

\section{Transfected Cell Lines}

GPE86 RV-packaging cells were transfected with the construct pLNChG65T (Figure 1). Once selected, nearly $100 \%$ of the cells emitted green fluorescence after excitation (data not shown). No cytopathic or growth-inhibiting effects due to the expression of pLNChG65T in transfected cells were observed.

\section{Transduced Expression of GFP}

The LNChG65T vector-producer cell line (VPC) exhibited intense fluorescence (Figure 2A). A375LNChG65T-transduced cells (Figure 2B), examined $72 \mathrm{~h}$ after transduction, demonstrated fluorescence in approximately $50 \%$ of the cells. After 7 days of G-418 selection, approximately $90 \%$ of 
the A375 (data not shown) and $70 \%$ of the IGROV cells demonstrated fluorescent activity (Figure 2C). By 14 days after selection, both of the transduced cell lines demonstrated nearly $100 \%$ fluorescence. No significant background fluorescence was detected in nontransduced cells (10).

\section{FACS Analysis of GFP-Transduced Cell Lines}

PA317, A375 and IGROV cells transduced by the LNChG65T RV vector and selected in G-418 were analyzed by FACS. The PA317- and A375transduced cells (Figure 3, B and D) were distinguished by up to a three log shift in mean fluorescent intensity in comparison to nontransduced control cells (Figure 3, A and C). Transduced IGROV cells (Figure 3F) also showed a significant shift in fluorescence intensity (Figure 3E).

\section{DISCUSSION}

The ability to observe gene activity in living cells is now optimized with the hGFP-S65T fluorescent gene marker system. Expression studies using the wild-type GFP gene in many eukaryotic cells and organisms have been hampered by the relatively low levels of fluorescence detected. We were not able to detect wild-type GFP gene product by either fluorescence microscopy or FACS in wild-type GFP RV-transduced and selected mammalians cells (10). Several modifications have been applied to improve the expression and fluorescence of GFP $(5-8,17)$. We have investigated several of these variants. In stable retroviral transduction with the red-shift mutation only, fluorescence has been reported (3), but it was at least one log less intense than pLNChRG-expressing cells (10). The "humanization" of 88 codons, without the red-shift mutation, in the GFP open reading frame and subsequent expression in pLNCX showed only moderate levels of fluorescence by both microscopic and FACS analysis (unpublished observation). When we combined 88 codon modifications with the red-shift mutation, a substantial increase in fluores- cence was observed (10). In this report, we demonstrate a greater improvement using a more extensively modified GFP gene in the pLNChG65T vector. This hGFP-S65T gene contains 81 more codon modifications compared to the $h R G F P$ gene (10). This improvement is demonstrated by comparing the $79.9 \%$ shift in fluorescence in the hRGFP VPC (10), to the $95.8 \%$ with the current version (Figure 3B). Furthermore, Southern analysis of an individually selected, highly fluorescent clonal population of the A375-hGFPS65T cells revealed the integration of one copy of the $h G F P$-S65T gene (data not shown), establishing the remarkable sensitivity of this system. Undoubtedly, further modifications will lead to continued development of GFP transgene fluorescence, such as that reported recently by Cormack et al. (5). It is possible that specific GFP variants will be most useful in certain mammalian cell lines or with specific gene transfer vectors. Direct comparison of each available GFP is currently in progress to assess the contribution of each improvement in a retroviral system. The $h G F P$-S65T GFP gene will allow researchers to see real-time events in living cells, tissues and animals and will likely revolutionize the study of gene expression.

\section{ACKNOWLEDGMENTS}

We thank Jeannie Malatesta, Ginger Dreifurst and Julie Seiwert for technical assistance with the flow cytometry.

\section{REFERENCES}

1.Amsterdam, A., S. Lin and N. Hopkins. 1995. The Aequorea victoria green fluorescent protein can be used as a reporter in live zebrafish. Dev. Biol. 171:123-129.

2.Baulcombe, D.C., S. Chapman and S. Santa Cruz. 1995. Jellyfish green fluorescent protein as a reporter for virus infections. Plant $\mathrm{J}$. 7:1045-1053

3.Cheng, L., J. Fu, A. Tsukamoto and R.G. Hawley. 1996. Use of green fluorescent protein variants to monitor gene transfer and expression in mammalian cells. Nature Biotechnol. 14:606-609.

4.Cone, R.D. and R.C. Mulligan. 1984. Highefficiency gene transfer into mammalian cells: generation of helper-free recombinant retrovirus with broad mammalian host range. Proc. Natl. Acad. Sci. USA 81:6349-6353.

5.Cormack, B.P., R.H. Valdivia and S.
Falkow. 1996. FACS-optimized mutants of the green fluorescent protein (GFP). Gene 173:33-38.

6.Crameri, A., E.A. Whitehorn, E. Tate and W.P.C. Stemmer. 1996. Improved green fluorescent protein by molecular evolution using DNA shuffling. Nature Biotechnol. 14:315319.

7.Haas, J., E.C. Park and B. Seed. 1996. Codon usage limitation in the expression of HIV-I envelope glycoprotein. Curr. Biol. 6:315-324.

8.Heim, R., A.B. Cubitt and R.Y. Tsien. 1995. Improved green fluorescence. Nature 373: 663-664.

9.Ikawa, M., K. Kominami, Y. Yoshimura, K. Tanaka, Y. Nishimune and M. Okabe. 1995. Green fluorescent protein as a marker in transgenic mice. Dev. Growth Differ. 37:455-459.

10.Levy, J.P., R.R. Muldoon, S. Zolotukin and C.J. Link. 1996. Retroviral transfer and expression of humanized, red shifted green fluorescent protein into human tumor cells. Nature Biotechnol. 14:610-614.

11.Link, C.J. and E.J. Beecham. 1994. Human gene therapy trials for cancer. J Biotech. Healthcare 2:183-195.

12.Miller, A.D. and C. Buttimore. 1986. Redesign of retrovirus packaging cell lines to avoid recombination leading to helper virus production. Mol. Cell Biol. 6:2895-2902.

13.Prasher, D.C. 1995. Using GFP to see the light. Trends Genet. 11:320-323.

14.Prasher, D.C., V.K. Eckenrode, W.W. Ward, F.G. Prendergrast and M.J. Cormier. 1992. Primary structure of the Aequorea victoria green-fluorescent protein. Gene 111:229-233

15.Rosenberg, S.A., P. Aebersold, K. Cornetta, A. Kasid, R. Morgan, R. Moen, E. Karson, M.T. Lotze, J.C. Yang, et al. 1990. Gene transfer into humans-Immunotherapy of patients with advanced melanoma, using tumorinfiltrating lymphocytes modified by retroviral gene transduction. N. Engl. J. Med. 323:570-578.

16.Wang, S. and T. Hazelrigg. 1994. Implications for bcd mRNA localization from spatial distribution of exu protein in Drosophila oogenesis. Nature 369:400-403.

17.Zolotukhin, S., M. Potter, W.W. Hauswirth, J. Guy and N. Muzyczka. 1996. A "humanized" green fluorescent protein cDNA adapted for high level expression in mammalian cells. J. Virol. 70:4646-4654.

Received 14 February 1996; accepted 9 July 1996.

Address correspondence to:

Charles J. Link

Gene Therapy Program

HGTRI

1415 Woodland Ave.

Des Moines, IA 50309, USA 